\title{
Analysis and Synthesis of the Offset Slider- Elliptic Crank Dwell Mechanism
}

\author{
Jiguang $\mathrm{Han}^{1,}$ a, Zhenzhi He $\mathrm{He}^{1, \mathrm{~b}}$ \\ ${ }^{1}$ Jiangsu Normal University, Xuzhou, Jiangsu, China \\ ahjg@jsnu.edu.cn, bhezz82@163.com
}

\begin{abstract}
Keywords: Combination mechanism; Slider-crank; Ellipse; Mechanism synthesis; Dwell
Abstract. Elliptic crank is able to generate elliptic curve of the planet mechanism, with elliptic crank as the driven crank of slider-crank mechanism is equal to the mechanism which has a variable length and a variable speed along the elliptical moving crank, which changed the kinematic characteristics of the slider. T The offset slider elliptical crank mechanism can be realized in the dwell and quick return characteristics at the same time. In this paper the analysis and synthesis methods of the offset slider elliptic crank combined mechanism is proposed which has dwell characteristics at the left side limit position. The calculation method of the performance parameters of the mechanism is given such as minimum transmission Angle, time ratio, etc. The method of determining the parameters of the mechanism are studied on the basis of allowable transmission angle.
\end{abstract}

\section{Introduction}

Slider-crank mechanism is one of the common applied four-link mechanisms. In order to improve its kinetic characteristic, some combined mechanisms with elliptical gear have been studied by some papers, and some special kinetic rules have been obtained [1-4]. However, the manufacture difficulty of elliptical gear suppressed its application. As we know, the track of point $A$ on the planet gear in planetary mechanism, which is combined by a fixed center wheel $z_{5}$, a tie bar 1 and a planet wheel $z_{2}$, is a cycloid curve [5,6]. When the relative transmission ratio of planetary wheel $z_{2}$ and fixed center wheel $z_{5}$ relative to tie bar $O_{1} B$ (driving link $l$ ) is $i=z_{5} / z_{2}=2$, the cycloid curve will degrade into elliptic curve, as shown by dotted line in Fig. 1. This planetary mechanism can be named as elliptical crank or elliptical production mechanism.

Because the moving point $A$ have different radius and speed in different positions, we can use the moving point $A$ to replace the crank drive slider, which can change the moving rule and obtain the slider mechanism similar as intermittent motion, on the extreme positions [7]. Eccentric elliptical slider-crank mechanism not only can realize the intermittent movement at some extreme point, but also have the characteristic of quick-return movement.

In this paper, the analysis and synthetic approach of this mechanism has been proposed. According to the allowable transmission angle, the extreme values of planet tie bar length and offset distance have been obtained. Then the calculation method of some parameters has been proposed, such as minimum transmission angle, extreme positions, travel speed changeable coefficients, etc. At last, the influence of main parameters on the mechanism kinematic performance and mechanism parameters which has the maximum travel speed changeable coefficients have been analyzed.

\section{Mechanism kinematic analysis}

As shown in Fig.1, some parameters have been defined in order to be easy analyzed. The length of tie bar $O_{1} B$ is 1 . The relative length of connected $\operatorname{rod} B A$ is $b$. The relative length of connected rod $A C$ is $l$. The Relative eccentricity of the sliding block is $e$. The position of driving link is $\phi_{1}$. The planet connected rod position of $B A$ is $\phi_{2}$. At the initial moment when $\phi_{1}=0$, the position of planet connected rod is $\phi_{0}$, as shown in Fig. 1 using filament. 


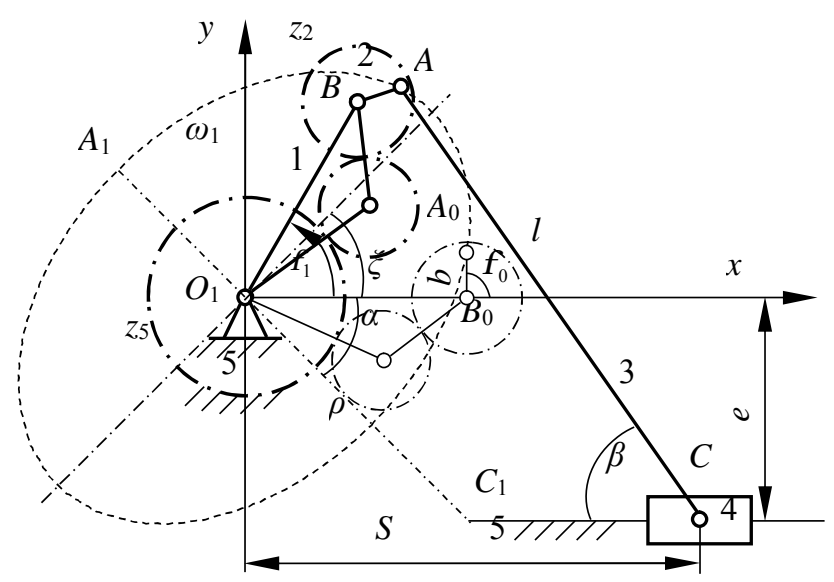

Fig.1. The ellipse crank slider block mechanism

According to principle of mechanism inversion, the relative transmission ratio $i_{25}^{1}$ is

$$
i_{25}^{1}=\frac{\omega_{2}-\omega_{1}}{\omega_{5}-\omega_{1}}=\frac{z_{5}}{z_{2}}=2=\frac{\mathrm{d} \phi_{2}-\mathrm{d} \phi_{1}}{0-\mathrm{d} \phi_{1}}
$$

Then can obtained $d \phi_{2}=-d \phi_{1}$. Do integration and then take initial condition $\phi_{1}=0, \phi_{2}=\phi_{0}$ together, and obtain

$$
\phi_{2}=\phi_{0}-\phi_{1}
$$

The coordinate of moving point $A$ is

$\left\{\begin{array}{l}x=\cos \phi_{1}+b \cos \left(\phi_{0}-\phi_{1}\right) \\ y=\sin \phi_{1}+b \sin \left(\phi_{0}-\phi_{1}\right)\end{array}\right.$

The included angle $\beta$ between connected $\operatorname{rod} A C$ and $\mathrm{X}$-axis is

$\beta=\arcsin \frac{y+e}{l}=\arcsin \frac{\sin \phi_{1}+b \sin \left(\phi_{0}-\phi_{1}\right)+e}{l}$

The displacement equation of the slid block is

$S=x+l \cos \beta$

The speed equation of the slid block can be obtained by derivation of time on equation (4)

$S^{\prime}=x^{\prime}-l \sin \beta \beta^{\prime}=x^{\prime}-(y+e) \beta^{\prime}$

Where, $x^{\prime}$ and $\beta^{\prime}$ can be obtained by derivation of time on equation (2) and equation (3)

$$
\begin{aligned}
& x^{\prime}=\left[-\sin \phi_{1}+b \sin \left(\phi_{0}-\phi_{1}\right)\right] \omega_{1}, y^{\prime}=\left[\cos \phi_{1}-b \cos \left(\phi_{0}-\phi_{1}\right)\right] \omega_{1} \\
& \beta^{\prime}=\frac{y^{\prime}}{l \cos \beta}=\frac{\left[\cos \phi_{1}-b \cos \left(\phi_{0}-\phi_{1}\right)\right] \omega_{1}}{\sqrt{l^{2}-\left(\sin \phi_{1}+b \sin \left(\phi_{0}-\phi_{1}\right)+e\right)^{2}}}
\end{aligned}
$$

The accelerated velocity equation of slid block can be obtained by time derivation to equation (5)

$S^{\prime \prime}=x^{\prime \prime}-y^{\prime} \beta^{\prime}-(y+e) \beta^{\prime \prime}$

Where, $x^{\prime \prime}$ and $\beta^{\prime \prime}$ can be obtained by 2-order derivation to equation (2) and equation (3)

$x^{\prime \prime}=-\left[\cos \phi_{1}+b \cos \left(\phi_{0}-\phi_{1}\right)\right] \omega_{1}^{2}, y^{\prime \prime}=-\left[\sin \phi_{1}+b \sin \left(\phi_{0}-\phi_{1}\right)\right] \omega_{1}^{2}$

$\beta^{\prime \prime}=\beta^{\prime 2} \operatorname{tg} \beta+\frac{y^{\prime \prime}}{l \cos \beta}$

The jerk equation of slid block can be obtained by time derivation to equation (6)

$S^{\prime \prime \prime}=x^{\prime \prime \prime}-y^{\prime \prime} \beta^{\prime}-2 y^{\prime} \beta^{\prime \prime}-(y+e) \beta^{\prime \prime \prime}$ 
Where, $x^{\prime \prime \prime}$ and $\beta^{\prime \prime \prime}$ can be obtained by 3 -order derivation to equation (2) and equation (3)

$$
\begin{aligned}
& x^{\prime \prime \prime}=\left[\sin \phi_{1}-b \sin \left(\phi_{0}-\phi_{1}\right)\right] \omega_{1}^{3}, y^{\prime \prime \prime}=\left[-\cos \phi_{1}+b \cos \left(\phi_{0}-\phi_{1}\right)\right] \omega_{1}^{3} \\
& \beta^{\prime \prime \prime}=3 \beta^{\prime} \beta^{\prime \prime} \operatorname{tg} \beta+\beta^{\prime 3}+\frac{y^{\prime \prime \prime}}{l \cos \beta}
\end{aligned}
$$

The movement curve of the slider elliptical crank mechanism is shown in Fig.2. The slider block 4 in this mechanism only have the moving value of 0.005 in the range of $\phi_{1}=120.39^{\circ} \sim 180.47^{\circ}$. This range can be seen as intermittent range, can be defined as $\phi_{\mathrm{d}}$. It can be seen from the movement curve that, the characteristic of the intermittent position $A_{1}$ is that, all the value of speed $S^{\prime}$, acceleration $S^{\prime \prime}$ and jerk $S^{\prime \prime \prime}$ are 0 .

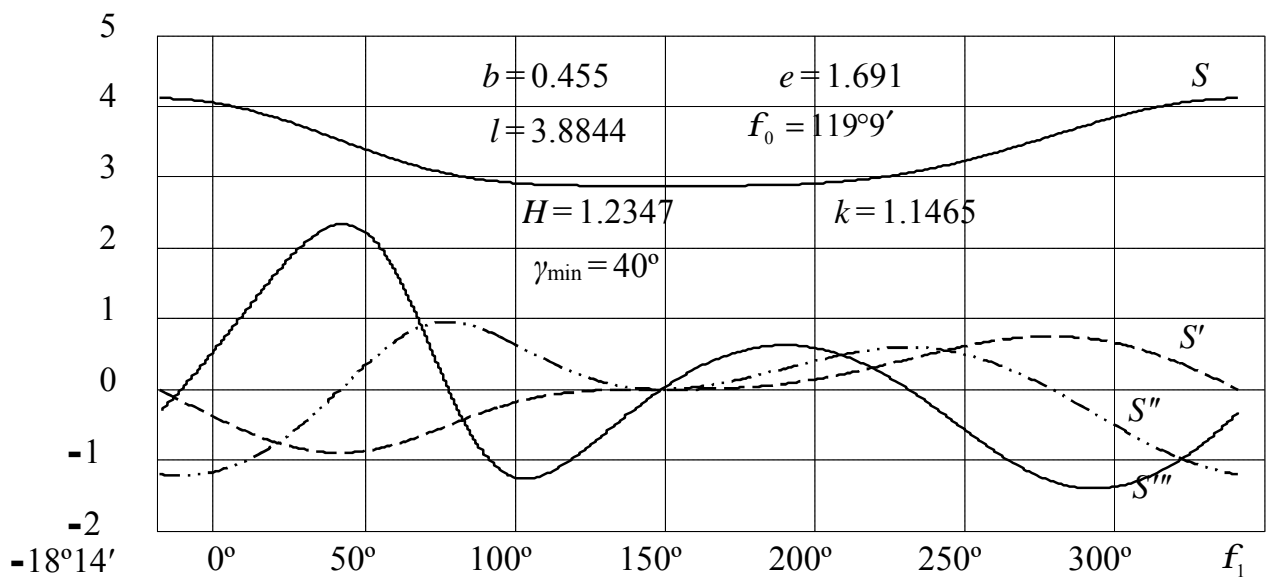

Fig. 2. The movement curve of the slider elliptical crank mechanism

\section{Mechanism synthesis}

Basic parameter relationship. There are totally 4 main parameters of slider elliptical crank mechanism, which are $b 、 e 、 l$ and $\phi_{0}$, as shown in Fig. 1. If the slider block has the condition of intermittent on the left extreme position $C_{1}$, then we can obtain that the position of $C_{1}$ is on the curvature center of point $A_{1}$ on ellipse minor axis. The length axis radius of elliptic is $1+b$, the short axis radius is $1-b$. Then the curvature radius $\rho$ of $A_{1}$ is

$$
\rho=\frac{(1+b)^{2}}{1-b}=l
$$

Along with the increasing of parameter $b, l$ will increase quickly, which will lead to the big size of mechanism. Therefore, the parameter $b$ should be better less than 0.6 .

The distance between the crank rotor center $O_{1}$ and slider block left extreme position $C_{1}$ is

$O_{1} C_{1}=\rho-(1-b)=\frac{4 b}{1-b}$

The intersection angle between ellipse short axis and $\mathrm{X}$-axis named $\alpha$ is

$$
\alpha=\arcsin \frac{e}{O_{1} C_{1}}=\arcsin \frac{e(1-b)}{4 b}
$$

The position of the long axis is $\phi_{1}=\phi_{2}=\xi$. Then we can obtain from this and equation (1) $\xi=\frac{\phi_{0}}{2}=\frac{\pi}{2}-\alpha$

It shows that, the function of initial position angle $\phi_{0}$ is to rotate the ellipse long axis from the $\mathrm{X}$-axis to the $\phi_{0} / 2$ angle on the anticlockwise direction. Combined with equation (8) and the initial position angle $\phi_{0}$ will be calculated 


$$
\phi_{0}=\pi-2 \arcsin \frac{e(1-b)}{4 b}
$$

Specially, when the parameter $e=0, \phi_{0}=\pi$, is a constant.

Equation (8) and equation (10) shows that, only 2 of the 4 parameters of the mechanism are independent. After choosing the parameter $b, l$ will be confirmed. Then after confirm parameter $e$, the initial position angle $\phi_{0}$ will be confirmed.

Minimum driving angle $\gamma_{\text {min }}$. The driving angle $\gamma$ and $\beta$ are complementary angles. The minimum driving angle $\gamma_{\min }$ will be appeared on the moment when $y$ is the maximum, i.e, $y^{\prime}=0$. It will be obtained from equation (5) that $\cos \phi_{1}-b \cos \left(\phi_{0}-\phi_{1}\right)=0$

Then

$$
\phi_{1}=\phi_{\min }=\operatorname{arctg} \frac{1-b \cos \phi_{0}}{b \sin \phi_{0}}
$$

The minimum driving angle $\gamma_{\min }$ is

$$
\gamma_{\min }=\frac{\pi}{2}-\arcsin \frac{\sin \phi_{\min }+b \sin \left(\phi_{0}-\phi_{\min }\right)+e}{l}
$$

Maximum offset $\boldsymbol{e}_{\max }$. Define the allowable driving angle is $[\gamma]$. The driving angle $\gamma$ should satisfy $\gamma_{\min } \geq[\gamma]$, that is to say, $\cos \gamma_{\min } \leq \cos [\gamma]$.

From equation (12)

$$
\cos \gamma_{\min }=\frac{\sin \phi_{\min }+b \sin \left(\phi_{0}-\phi_{\min }\right)+e}{l} \leq \cos [\gamma]
$$

After some conversion the driving angle equation will be obtained

$$
\begin{aligned}
& \cos \phi_{\min }\left(1+b^{2}-2 b \cos \phi_{0}\right)+b(e-l \cos [\gamma]) \sin \phi_{0} \leq 0 \text { From equation (10) we will obtain } \\
& \cos \phi_{0}=\frac{e^{2}(1-b)^{2}}{8 b^{2}}-1 \\
& \sin \phi_{0}=\frac{e(1-b) \sqrt{16 b^{2}-e^{2}(1-b)^{2}}}{8 b^{2}}
\end{aligned}
$$

From equation (11) we will obtain

$$
\cos \phi_{\min }=\frac{e(1-b) \sqrt{16 b^{2}-e^{2}(1-b)^{2}}}{4 \sqrt{4 b^{2}(b+1)^{2}-e^{2} b(1-b)^{2}}}
$$

Combine the above 4 equations and simplify the results

$$
b(1-b)^{2} e^{2}-8 b^{2}(1-b) \cos [\gamma] e+4\{b(1+b) \cos [\gamma]\}^{2}+4 b^{2}(1-b)^{2} \leq 0
$$

then

$$
e \leq \frac{4 b \cos [\gamma]}{1-b}-2 \sqrt{b} \sin [\gamma]=e_{\max }
$$

The relationship between the maximum offset $\mathrm{e}_{\max }$, the planet connecting rod length $b$, and the allowable driving angle $[\gamma]$ is shown in Fig.3.

Minimum connecting rod length $\boldsymbol{b}_{\text {min }}$. The offset $e$ must bigger or equal 0 . From equation (13) we can obtain $\operatorname{tg}^{2}[\gamma] b^{2}-2\left(\operatorname{tg}^{2}[\gamma]+2\right) b+\operatorname{tg}^{2}[\gamma] \leq 0$

Then

$$
\frac{1+\cos [\gamma]}{1-\cos [\gamma]} \geq b \geq \frac{1-\cos [\gamma]}{1+\cos [\gamma]}
$$

Because the parameter $b<1$, then the maximum value restriction of $b$ in the above equation will be satisfied naturally. Then 


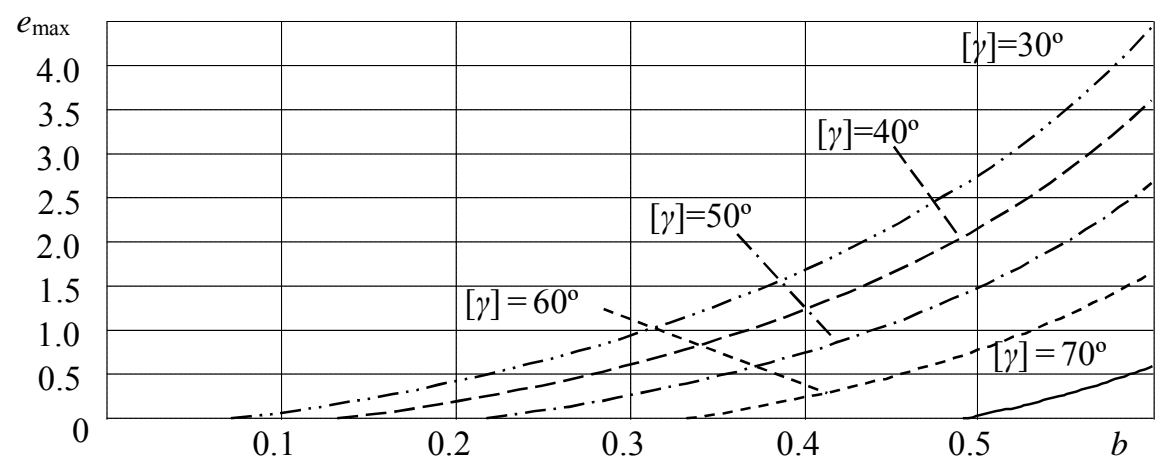

Fig. 3. The relationship between $e_{\max }, b$ and $[\gamma]$

$b \geq \frac{1-\cos [\gamma]}{1+\cos [\gamma]}=b_{\min }$

The relationship between the minimum connecting rod length $b_{\min }$ and allowable driving angle $[\gamma]$ is shown in Fig. 4.

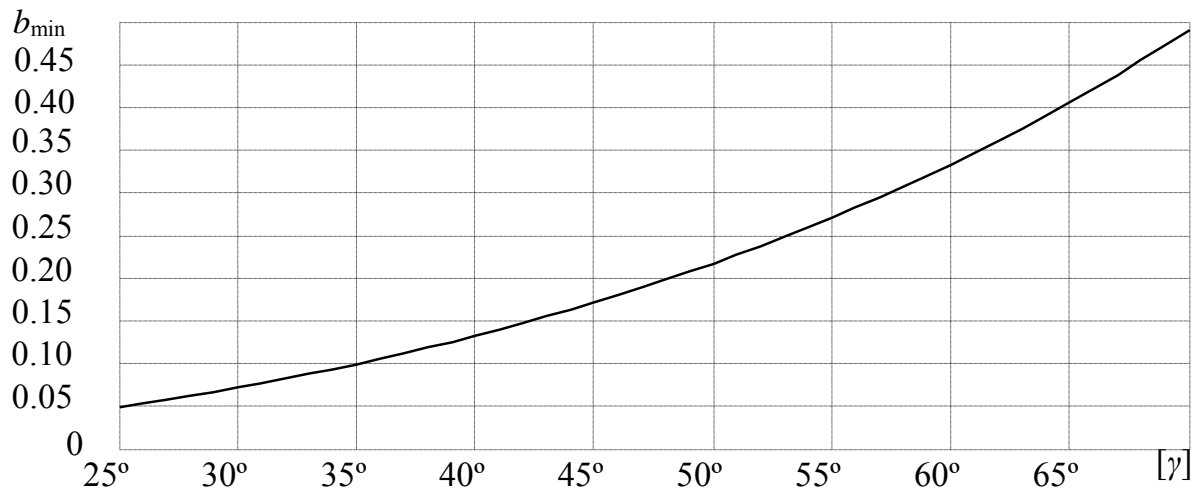

Fig. 4. The relationship between $b_{\min }$ and $[\gamma]$

Sliding block extreme position. The left extreme position $C_{1}$ of the slider block can be confirmed by Fig. 1 . The position $\phi_{11}$ of the $C_{1}$ corresponding original driving link 1 is

$\phi_{11}=\pi-\alpha=\pi-\arcsin \frac{e(1-b)}{4 b}$

The abscissa $S_{1}$ of $C_{1}$ is

$$
S_{1}=\sqrt{O_{1} C_{1}^{2}-e^{2}}=\frac{\sqrt{16 b^{2}-e^{2}(1-b)^{2}}}{(1-b)}
$$

It is difficult to calculate the right extreme position $C_{2}$ and its corresponding driving link 1 position $\phi_{12}$. Theoretically, the circle centered by $C_{2}$ and radial of connecting rod length $l$ should be contact of curve between the elliptic curve. At the contact point, both the ellipse and the circle should have the same tangent line, that is to say, the tangent line of ellipse is perpendicular to the line between contact point and circle center $C_{2}$. Then we can obtain

$\frac{y^{\prime}}{x^{\prime}}=-\frac{x-s}{y+e}$

Combined with equation (4) and (3)

$$
(y+e) y^{\prime}-x^{\prime} \sqrt{l^{2}-(y+e)^{2}}=0
$$


According to the extremum condition of slider block displacement $S$ that $S^{\prime}=0$, the equation (17) can also be obtained by equation (5). A 4-order equation can be obtained by equation (17) about $\cos \phi_{1}$, and at the same time have the cross term between $\sin \phi_{1}$. Actually, also the root is exist, it is very hard to calculate the root by ordinary algebra methods. The only way to calculate the root is the numerical method. Define the root of equation (17) is $\phi_{1}=\phi_{12}$, combine with the equation (2) (4) and calculate the left extreme position $S_{2}$ of the slider block. The relative route $H$ can be described by

$$
H=S_{2}-S_{1}=S_{2}-\frac{\sqrt{16 b^{2}-e^{2}(1-b)^{2}}}{(1-b)}
$$

The given real slider block route divide the relative route $H$, can obtain the original driving link real length. That is the magnification times of the other length factors $b, e$ and $l$.

The coefficient of travel speed variation $k$ can be expressed as

$$
\begin{aligned}
& k=\frac{\phi_{11}-\phi_{12}}{2 \pi-\phi_{11}+\phi_{12}}, \quad \phi_{11}-\phi_{12}>\pi \\
& k=\frac{2 \pi-\phi_{11}+\phi_{12}}{\phi_{11}-\phi_{12}}, \quad \phi_{11}-\phi_{12}<\pi
\end{aligned}
$$

\section{Conclusions}

The ellipse crank slider block mechanism can realize the interval movement at an extreme position. The bias ellipse crank slider block mechanism can at the same time realize the quick-return movement. The ellipse crank slider block mechanism only have 2 parameters can be chosen. Considering the structure size, we should choose the smaller planet connecting rod length $b$ and the bigger offset distance $e$.

\section{References}

[1] J IA NG Miao, CHE N J ian-neng, Z H AO Yun. Journal of Zhejiang Sci-Tech University Vol 26 (2009), p. 256-260(In Chinese)

[2] Zhou Jie, Zhou Shaohua. Journal of Wuhan Transportation University Vol 22 (1998), p. 304-307 (In Chinese)

[3] Gao Xueqiang, Yang Shengqiang,Ma Lin. Journal of Taiyuan University of technology Vol 33 (2002), p. 41-43(In Chinese)

[4] WANG Bo, ZHANG Mei-lin. Journal of Beijing university of chemical technology Vol 27 (2000), p. 60-62 (In Chinese)

[5] Han Jiguang, Wei Jiguang, Liu Shaogang. Forestry machinery and woodworking equipment Vol 25 (1997), p. 27-28 (In Chinese)

[6] Han Jiguang, Lu Zhonglv, Wu Ronggao. Journal of Jiamusi technology university Vol 9 (1991), p. 129-133 (In Chinese)

[7] Han Jiguang, Zhang Chuanyan, Zuo Weiyang. Analysis and Synthesis of the In-line Slider-Elliptic Crank Dwell Mechanism. International Journal of Control and Automation Vol 7 (2014), p. 233-242

[8] Zhang Yanbin, Wu Xin, Liu Hongzhao, Liu Huiyang. Transactions of Chinese society for agricultural machinery Vol 40 (2009), p. 195-198 (In Chinese)

[9] Zheng WenWei, etc. Mechanical principles (6th edition), Higher education press. 2007 